\title{
Evaluación de una solución inmovilizadora para criopreservación del semen de Colossoma macropomum, "Gamitana"
}

\section{[Evaluation of solution restraining for cryopreservation of sperm Colossoma macropomum "Gamitana"]}

\author{
Ehrlich Llasaca-Calizaya ${ }^{1}$, Lorgio Verdi-Olivares ${ }^{2}$, Jesús Nuñez-Rodriguez ${ }^{1,3}$ \\ ${ }^{1}$ Universidad Nacional de la Amazonía Peruana, Escuela de Post Grado, Cátedra CONCYTEC, Maestría en Acuicultura, \\ Iquitos, Perú. \\ Universidad Nacional de la Amazonía Peruana, Facultad de Ciencias Biológicas, Escuela de Acuicultura, Pevas $5^{\text {ta }}$ \\ Cuadra, Iquitos, Perú \\ ${ }^{3}$ Instituto de Investigaciones para el desarrollo, IRD-UR 175, Francia. \\ *e-mail: Ehrlich2@hotmail.com
}

\begin{abstract}
Resumen
El objetivo del trabajo fue la criopreservacion de semen, que permitirá constituir un banco genético, para lo cual se buscó obtener una solución inactivadora de colecta para el semen de Colossoma macropomum "gamitana", que permita obtener espermatozoides, con buena motilidad de activación después de la descongelación, en nitrógeno líquido. Se utilizó semen de reproductores mantenidos, del Instituto de Investigaciones de la Amazonía Peruana (IIAP) inducidos con Conceptal ${ }^{\circledR}$ y sin inducir mantenidos en el Centro de Acuicultura Nuevo Horizonte del Fondo Nacional de Desarrollo Pesquero (CANH - FONDEPES). El semen fue colectado en soluciones inactivadoras de $9 \%$ y $10 \%$ de $\mathrm{NaCl}$, añadiendo $2 \mathrm{~g} / \mathrm{L}, 4 \mathrm{~g} / \mathrm{L}$ y $8 \mathrm{~g} / \mathrm{L}$ de $\mathrm{NaHCO}_{3}$, soluciones de sacarosa (300 mM, $400 \mathrm{mM}$ y $500 \mathrm{mM}$ ) sola o con 1,5 g/L, $1 \mathrm{~g} / \mathrm{L} \mathrm{y} \mathrm{0,5} \mathrm{g/L} \mathrm{de} \mathrm{NaCl.}$ Se concluye que el tratamiento de $400 \mathrm{mM}$ de sacarosa dio el mejor resultado, con una motilidad del $80 \%$ y 40 segundos de duración. También se evaluó la motilidad, después de una hora de almacenamiento a temperatura ambiente, con $60 \%$ de motilidad después de la activación y 20 segundos de duración. Este trabajo permitirá desarrollar un protocolo de criopreservación para lotes de semen inmovilizados, con tiempo suficiente para preparar las pajuelas, congelarlas en nitrógeno líquido y optimizar el manejo de reproductores.
\end{abstract}

Palabras claves: Colossoma macropomum, criopreservación, motilidad, sacarosa, tiempo de activación

\begin{abstract}
The objective was the cryopreservation of semen, which enable a gene bank, for which a solution was sought inactivating semen collection for Colossoma macropomum "tambaqui" to obtain sperm with good motility after activation thawing in liquid nitrogen. The collection inactivating solution was prepared with semen from players held at the Institute of Amazonian Research (IIAP)-induced without inducing Conceptal ${ }^{\circledR}$ and maintained at the station of New Horizon National Fisheries Development Fund (FONDEPES). Semen was collected in solutions of $9 \%$ and $10 \% \mathrm{NaCl}$, adding $2 \mathrm{~g} / \mathrm{L}, 4 \mathrm{~g} / \mathrm{L}$ and $8 \mathrm{~g} / \mathrm{L} \mathrm{NaHCO}$. As inactivating solution was tested 3 concentrations of sucrose $(300 \mathrm{mM}, 400 \mathrm{mM}$ and $500 \mathrm{mM}$ ) alone or with $1.5 \mathrm{~g} / \mathrm{L}, 1 \mathrm{~g} / \mathrm{L}$ and $0.5 \mathrm{~g} / \mathrm{L} \mathrm{NaCl}$. The treatment of $400 \mathrm{mM}$ sucrose gave the best result, with $80 \%$ motility and 40 seconds long. Motility was also evaluated after one hour storage at room temperature with $60 \%$ motility after activation and 20 seconds long. This work will develop a protocol for cryopreservation of semen frozen batches, with sufficient time to prepare the straws, frozen in liquid nitrogen and optimize the management of players.
\end{abstract}

Keywords: Colossoma macropomum, cryopreservation, motility, sucrose, activation time. 


\section{INTRODUCCIÓN}

Colossoma macropomum "gamitana", es una especie muy requerida en la Amazonía. Por ser una especie migradora, presenta problemas, en la reproducción en cautiverio. Trayendo como consecuencia, la reducción del volumen de semen y una disminución en la calidad de esperma. Siendo necesario, la aplicación de hormonas para inducir la espermiación y puesta, para completar su ciclo reproductivo en condiciones de control artificial. (Nizio et al., 2011; Valdebenito, 2008 e Iñiguez \& Renno, 2004)

Son muchos los beneficios y los estudios de la criopreservación de semen y su utilización para la conservación de las especies. Sin embargo, presentan bajos índices de fertilidad, porque la mayoría de los protocolos estudiados han sido derivados de aquellos utilizados en especies marinas, particularmente salmónidos y ciprínidos. No pudiendo establecer, procesos prácticos que permitan el uso de ésta técnica a escala comercial. (Murgas et al., 2001; Liu, 2006; Martino, 2006; Otemé et al., 1996; Carolsfeld et al., 2003; Ribeiro \& Godinho, 2003; Chereguini et al., 1992; Leung y Jaieson, 1991). En general, los resultados son muy variables, indicando una alta sensibilidad de los protocolos propuestos, lo cual hace necesario, en la mayoría de los casos, ajustar el procedimiento para cada especie (krone y Wittbrodt, 1997; Ciereszko y Dabrowski, 1993).

Surge entonces la necesidad de realizar trabajos que permitan perfeccionar un protocolo para la crioconservación del semen de esta especie, que garantice adecuados índices de fertilidad y que permita aprovechar las ventajas que ofrece la inseminación artificial.

Los hallazgos de este trabajo constituyen un paso muy importante, orientado hacia el desarrollo de una tecnología que permita estandarizar el protocolo de criopreservación, para su uso a escala comercial.

\section{MATERIALES Y MÉTODOS \\ Lugar de Estudio}

El estudio se realizó en el Centro de Investigaciones de Quistococha (CIQ), sede del Programa de Investigación para el Uso y Conservación del Agua y sus Recursos
(AQUAREC) del IIAP. El CIQ está ubicado en el Km. 4.5 de la carretera Iquitos- Nauta en el distrito de San Juan Bautista, provincia de Maynas, Región Loreto. Y también, en las instalaciones del Centro de Acuicultura Nuevo Horizonte - $\mathrm{CANH}$, del Fondo Nacional de Desarrollo Pesquero - FONDEPES, ubicado en el km 40, de la carretera Iquitos - Nauta.

\section{Material Biológico}

Se trabajó con cuatro ejemplares de reproductores machos de gamitana, con un rango de peso promedio de $5 \mathrm{~kg}$ a $6 \mathrm{~kg}$, con una edad de 5 años aproximadamente, provenientes del IIAP, inducidos con Conceptal ${ }^{\circledR}$ y uno y sin inducir, mantenidos en el Centro de Acuicultura Nuevo Horizonte (Fondo Nacional de Desarrollo Pesquero), los cuales fueron seleccionados de acuerdo a las características externas de un ejemplar que ha alcanzado madurez sexual, establecido por Alcantara et al., 2002 (figura 1).

\section{Colecta y evaluación del semen}

Para la realización de los experimentos, se procedió a colectar semen de reproductores de gamitana (inducidos y sin inducir), mediante ligera presión abdominal, esperando que primero salga la orina y dejando que se pierda un poco de semen, para minimizar la contaminación de la muestra con orina, lo cual activaría la motilidad, del semen (figura 2). Luego se colectó en una cucharita o placa Petri, la muestra de semen, para luego observar en el microscopio una pequeña muestra del semen colectado y comprobar la inactivación de los espermatozoides. Posteriormente se procedió a activar la muestra observada al microscopio, con agua destilada, para comprobar la viabilidad de los espermatozoides.

Para la evaluación del semen, se realizó por observación directa del campo visual del microscopio y fue expresado en porcentaje aproximado de motilidad de los espermatozoides observados, tomando como referencia el momento inicial de la activación con agua destilada.

Para la evaluación del tiempo de almacenamiento de semen en solución de sacarosa, se consideró el tiempo que duró la motilidad de los espermatozoides.

Ensayos Experimentales 
El trabajo utilizó 4 reproductores machos y se desarrolló en cuatro ensayos experimentales, para las observaciones de los espermatozoides, se realizó de manera visual del campo óptico, del microscopio. Fueron realizados 4 ensayos experimentales en CIQ y uno en el CANH y un ensayo para evaluar el tiempo de almacenamiento corto en solución de sacarosa.

Ensayo experimental 1: $\mathrm{NaCl}$ y Bicarbonato de Sodio, para el semen de 3 reproductores del CIQ, inducidos con Conceptal ${ }^{\circledR}$.

Se evaluaron 3 reproductores machos, inducidos con Conceptal ${ }^{\circledR}$, utilizando soluciones de $9 \%$ y $10 \%$ de $\mathrm{NaCl}$, añadiendo $2 \mathrm{~g} / \mathrm{L}, 4 \mathrm{~g} / \mathrm{L}$ y $8 \mathrm{~g} / \mathrm{L}$ de $\mathrm{NaHCO}_{3}$

Ensayo experimental 2, 3, 4, 5 y 6: $\mathrm{NaCl}$ y Sacarosa, para semen del reproductor del CIQ, inducido con Conceptal ${ }^{\circledR}$, para semen testicular, optimización de soluciones, sin inducir del CANH y almacenamiento de semen en solución de Sacarosa, por una hora a temperatura ambiente.

Se evaluó semen de un reproductor inducido con Conceptal ${ }^{\circledR}$, semen testicular inducido con Conceptal ${ }^{\circledR}$ y semen de un reproductor sin inducir del $\mathrm{CANH}$, en soluciones de 300 $\mathrm{mM}, 400 \mathrm{mM}$ y $500 \mathrm{mM}$ de Sacarosa sola o con $1,5 \mathrm{~g} / \mathrm{L}, 1 \mathrm{~g} / \mathrm{L}$ y $0,5 \mathrm{~g} / \mathrm{L}$ de $\mathrm{NaCl}$.

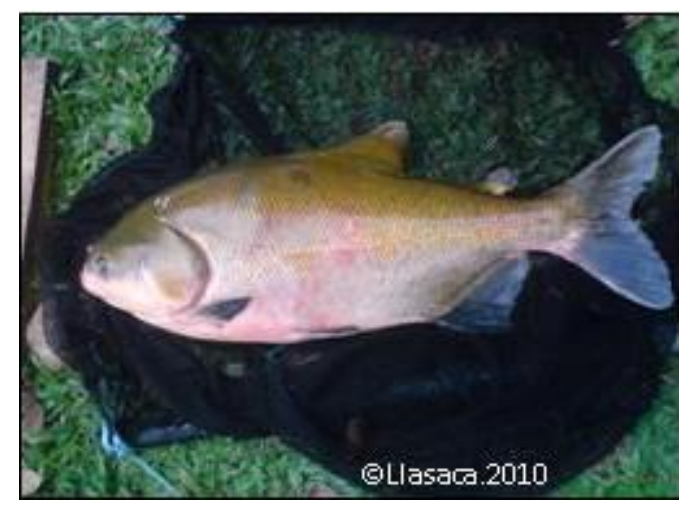

Figura 1. Reproductor de Colossoma macropomum "gamitana"

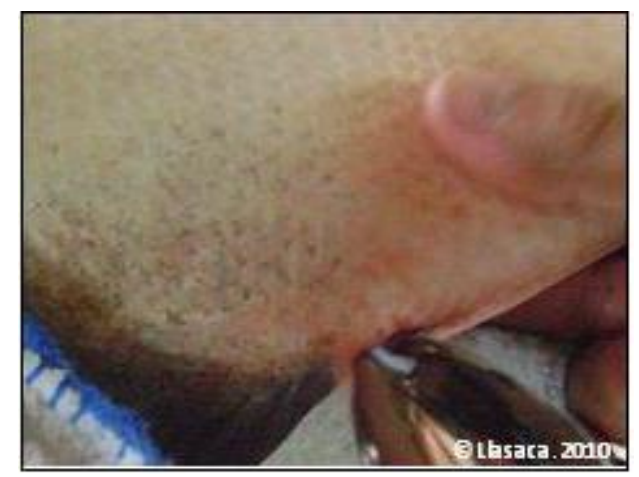

Figura 2. Colecta de semen de reproductor de Colossoma macropomun "gamitana" sin inducir 


\section{RESULTADOS}

Ensayo experimental 1: $\mathrm{NaCl}$ y Bicarbonato de Sodio, para el semen de 3 reproductores del CIQ, inducidos con Conceptal ${ }^{\circledR}$.

En la tabla 1, se puede observar, que ninguna solución evaluada, puede mantener inactivo el semen colectado, por presión abdominal.

Ensayo experimental 2: Evaluación de soluciones de $\mathrm{NaCl}$, Sacarosa y sus combinaciones, para semen del reproductor del CIQ, inducido con Conceptal ${ }^{\circledR}$.

En la tabla 2, se puede observar que sólo la solución de Sacarosa en un rango de concentración de 333 mM a 500 mM, es la que mejor inactivación de espermatozoides presenta, comparado a las demás concentraciones de soluciones. Manteniendo intactas sus condiciones de motilidad a diferentes tiempos de activación.

Ensayo experimental 3: Evaluación de soluciones de $\mathrm{NaCl}$, Sacarosa y sus combinaciones, para semen testicular, inducido con Conceptal ${ }^{\circledR}$

Los valores obtenidos, demuestran que para semen testicular, el rango de concentración de sacarosa es de 300 mM y 500 mM, también mantienen a los espermatozoides inmótiles (tabla 3).

Ensayo experimental 4: Optimización de soluciones de $\mathrm{NaCl}$, Sacarosa y sus combinaciones, para semen testicular, inducido con Conceptal ${ }^{\circledR}$

Según los valores obtenidos, se aprecia que niveles inferiores a $150 \mathrm{mM}$ de $\mathrm{NaCl}$, inician la activación de la motilidad en los espermatozoides. Sin embargo

Ensayo experimental 5: Evaluación de soluciones de Sacarosa, $\mathrm{NaCl} y$ sus combinaciones, para semen del reproductor del $\mathrm{CANH}$, sin inducir.

Se observa en la Tabla 5, que los mejores valores de activación con agua destilada (motilidad) se aprecian, en la solución de sucrosa a concentración de $400 \mathrm{mM}$, para semen colectado sin inducir.

Ensayo experimental 6: Evaluación de soluciones de Sacarosa, $\mathrm{NaCl} y$ sus combinaciones, para semen del reproductor del $\mathrm{CANH}$, sin inducir, después de una hora almacenado a temperatura ambiente (tabla $6)$.

Tabla 1. Valores de motilidad para semen de reproductor inducido con Conceptal ${ }^{\circledR}$, para soluciones de $9 \%$ y $10 \%$ de $\mathrm{NaCl}$, añadiendo $2 \mathrm{~g} / \mathrm{L}, 4 \mathrm{~g} / \mathrm{L}$ y $8 \mathrm{~g} / \mathrm{L}$ de $\mathrm{NaHCO}_{3}$.

\begin{tabular}{ccccccc}
\hline Solución & \multicolumn{2}{c}{ Reproductor 1 } & \multicolumn{2}{c}{ Reproductor 2 } & \multicolumn{2}{c}{ Reproductor 3 } \\
\cline { 2 - 6 } & $\begin{array}{c}\text { Motilidad } \\
\text { de Colecta } \\
\mathrm{s} / \mathrm{H}_{2} \mathrm{O}\end{array}$ & $\begin{array}{c}\text { Motilidad de } \\
\text { post Colecta } \\
\mathrm{c} / \mathrm{H}_{2} \mathrm{O}\end{array}$ & $\begin{array}{c}\text { Motilidad } \\
\text { de Colecta } \\
\mathrm{s} / \mathrm{H}_{2} \mathrm{O}\end{array}$ & $\begin{array}{c}\text { Motilidad de } \\
\text { post Colecta } \\
\mathrm{c} / \mathrm{H}_{2} \mathrm{O}\end{array}$ & $\begin{array}{c}\text { Motilidad } \\
\text { de Colecta } \\
\mathrm{s} / \mathrm{H}_{2} \mathrm{O}\end{array}$ & $\begin{array}{c}\text { Motilidad de } \\
\text { post Colecta } \\
\mathrm{c} / \mathrm{H}_{2} \mathrm{O}\end{array}$ \\
\hline $9 \%$ & 0,00 & 0,00 & 50,00 & 20,00 & 0,00 & 0,00 \\
$9 \%$ y 2 g & 20,00 & 20,00 & 70,00 & 0,00 & 20,00 & 0,00 \\
$9 \%$ y 4 g & 20,00 & 20,00 & 0,00 & 0,00 & 50,00 & 0,00 \\
$10 \%$ & 50,00 & 50,00 & 50,00 & 0,00 & 70,00 & 0,00 \\
$10 \%$ y $2 \mathrm{~g}$ & 0,00 & 0,00 & 0,00 & 0,00 & 0,00 & 0,00 \\
$10 \%$ y $4 \mathrm{~g}$ & 0,00 & 0,00 & 0,00 & 0,00 & 0,00 & 0,00 \\
$10 \%$ y $8 \mathrm{~g}$ & 0,00 & 50,00 & 20,00 & 0,00 & 0,00 & 0,00 \\
\hline
\end{tabular}

Tabla 2. Valores de motilidad para semen de reproductor inducido con Conceptal ${ }^{\circledR}$, para soluciones a diferentes $\mathrm{mM}$ de $\mathrm{NaCl}$, Sacarosa y sus combinaciones.

\begin{tabular}{cccccccc}
\hline Solución & Concentración & \multicolumn{6}{c}{ Motilidad* (\%) } \\
\cline { 3 - 7 } & & \multicolumn{2}{c}{$5 \mathrm{~min}}$. & \multicolumn{2}{c}{$20 \mathrm{~min}}$. & $\mathrm{min}$. \\
& $\mathrm{NaCl}(\mathrm{mM})$ & $\mathrm{s} / \mathrm{H}_{2} \mathrm{O}$ & $\mathrm{c} / \mathrm{H}_{2} \mathrm{O}$ & $\mathrm{s} / \mathrm{H}_{2} \mathrm{O}$ & $\mathrm{c} / \mathrm{H}_{2} \mathrm{O}$ & $\mathrm{s} / \mathrm{H}_{2} \mathrm{O}$ & $\mathrm{c} / \mathrm{H}_{2} \mathrm{O}$ \\
& & Destilada & Destilada & Destilada & Destilada & Destilada & Destilada \\
$\mathrm{A}$ & 125 & 20,0 & 0,0 & 0,0 & 0,0 & 0,0 & 0,0 \\
$\mathrm{~B}$ & 250 & 0,0 & 0,0 & 0,0 & 0,0 & 0,0 & 0,0 \\
\hline
\end{tabular}




\begin{tabular}{|c|c|c|c|c|c|c|c|c|}
\hline $\mathrm{C}$ & \multicolumn{2}{|c|}{333} & 70,0 & 70,0 & 0,0 & 0,0 & 0,0 & 0,0 \\
\hline $\mathrm{D}$ & \multicolumn{2}{|c|}{500} & 0,0 & 0,0 & 0,0 & 0,0 & 0,0 & 0,0 \\
\hline \multirow[t]{2}{*}{$E$} & \multicolumn{2}{|c|}{1000} & 0,0 & 0,0 & 0,0 & 0,0 & 0,0 & 0,0 \\
\hline & \multicolumn{2}{|c|}{ Sacarosa (mM) } & \multicolumn{2}{|c|}{$5 \mathrm{~min}$} & \multicolumn{2}{|c|}{$20 \mathrm{~min}$} & \multicolumn{2}{|c|}{$30 \mathrm{~min}$} \\
\hline I & \multicolumn{2}{|c|}{125} & 0,00 & 0,00 & 0,00 & 0,00 & 0,00 & 0,00 \\
\hline II & \multicolumn{2}{|c|}{250} & 0,00 & 0,00 & 0,00 & 0,00 & 0,00 & 0,00 \\
\hline III & \multicolumn{2}{|c|}{333} & 15,00 & 60,00 & 0,00 & 50,00 & 0,00 & 40,00 \\
\hline IV & \multicolumn{2}{|c|}{500} & 0,00 & 50,00 & 0,00 & 30,00 & 0,00 & 30,00 \\
\hline \multirow[t]{2}{*}{$\mathrm{V}$} & \multicolumn{2}{|c|}{1000} & 0,00 & 0,00 & 0,00 & 0,00 & 0,00 & 0,00 \\
\hline & $\begin{array}{l}\mathrm{NaCl} \\
(\mathrm{mM})\end{array}$ & $\begin{array}{l}\text { Sacarosa } \\
(\mathrm{mM})\end{array}$ & \multicolumn{2}{|c|}{$5 \mathrm{~min}$} & \multicolumn{2}{|c|}{$20 \mathrm{~min}}$, & \multicolumn{2}{|c|}{$30 \mathrm{~min}$} \\
\hline 1 & 63 & 63 & 0,00 & 0,00 & 0,00 & 0,00 & 0,00 & 0,00 \\
\hline 2 & 125 & 125 & 0,00 & 0,00 & 0,00 & 0,00 & 0,00 & 0,00 \\
\hline 3 & 167 & 167 & 70,00 & 70,00 & 0,00 & 0,00 & 0,00 & 0,00 \\
\hline 4 & 250 & 250 & 0,00 & 20,00 & 0,00 & 0,00 & 0,00 & 0,00 \\
\hline 5 & 500 & 500 & 0,00 & 0,00 & 0,00 & 0,00 & 0,00 & 0,00 \\
\hline
\end{tabular}

*Motilidad activada, con agua destilada a diferentes tiempos.

Tabla 3. Valores de motilidad para soluciones a diferentes concentraciones de molaridad, de $\mathrm{NaCl}$, Sacarosa y sus combinaciones, para semen testicular, de reproductor inducido con Conceptal ${ }^{\circledR}$

\begin{tabular}{cccc}
\hline & Solución & \multicolumn{2}{c}{ Motilidad (\%)* } \\
\cline { 3 - 4 } No & $\begin{array}{c}\mathrm{s} / \mathrm{H}_{2} \mathrm{O} \\
\text { Destilada }\end{array}$ & $\begin{array}{c}\mathrm{c} / \mathrm{H}_{2} \mathrm{O} \\
\text { Destilada }\end{array}$ \\
\hline 1 & $300 \mathrm{mM}$ de Na Cl & 80 & 80 \\
2 & $500 \mathrm{mM}$ de NaCl & 5 & 80 \\
3 & $300 \mathrm{mM}$ de Sacarosa. & 0 & 80 \\
4 & $500 \mathrm{mM}$ de Sacarosa. & 0 & 40 \\
5 & $150 \mathrm{mM}$ de Sacarosa y 150 mM de NaCl & 70 & 70 \\
6 & $250 \mathrm{mM}$ de Sacarosa y 250 mM de NaCl. & 0 & 80 \\
\hline
\end{tabular}

*Motilidad evaluada a los 5 minutos de colectada la muestra.

Tabla 4. Valores de motilidad para la optimización de soluciones a diferentes combinaciones de concentraciones de molaridad, de $\mathrm{NaCl}$, Sacarosa, para semen testicular, de reproductor inducido con Conceptal ${ }^{\circledR}$

\begin{tabular}{lcc}
\hline No & Soluciónes (mM) & \multicolumn{2}{c}{ Motilidad* (\%) } \\
\cline { 2 - 3 } & $\mathrm{s} / \mathrm{H}_{2} \mathrm{O}$ & $\mathrm{c} / \mathrm{H}_{2} \mathrm{O}$ \\
Destilada & Destilada \\
\hline
\end{tabular}




\begin{tabular}{cccc}
\hline 1 & 300 Sacarosa y $150 \mathrm{NaCl}$ & $0 \%$ & $80 \%$ \\
2 & 300 Sacarosa y $100 \mathrm{NaCl}$ & $10 \%$ & $60 \%$ \\
3 & 300 Sacarosa y $50 \mathrm{NaCl}$ & $5 \%$ & $80 \%$ \\
\hline
\end{tabular}

${ }^{*}$ Motilidad evaluada a los 5 minutos de colectada la muestra. 
Tabla 5. Valores de motilidad para la Evaluación de soluciones de Sacarosa, $\mathrm{NaCl}$ y sus combinaciones, para semen del reproductor del CANH, sin inducir.

\begin{tabular}{|c|c|c|c|c|c|c|c|c|c|c|}
\hline \multirow[t]{2}{*}{ No } & \multicolumn{2}{|c|}{ Solución } & \multicolumn{2}{|c|}{ Replica 1} & \multicolumn{2}{|c|}{ Réplica 2} & \multicolumn{2}{|c|}{ Réplica 3} & \multicolumn{2}{|c|}{ Réplica 4} \\
\hline & $\begin{array}{c}\text { Sacarosa } \\
(\mathrm{mM}) \\
\mathrm{s} / \mathrm{H}_{2} \mathrm{O} \\
\text { Destilada }\end{array}$ & $\begin{array}{c}\mathrm{NaCl}(\mathrm{mM}) \\
\mathrm{c} / \mathrm{H}_{2} \mathrm{O} \\
\text { Destilada }\end{array}$ & $\begin{array}{l}\text { Motilidad de } \\
\text { Colecta }\end{array}$ & $\begin{array}{l}\text { Activación de } \\
\text { Motilidad }\end{array}$ & $\begin{array}{l}\text { Motilidad de } \\
\text { Colecta }\end{array}$ & $\begin{array}{l}\text { Activación de } \\
\text { Motilidad }\end{array}$ & $\begin{array}{l}\text { Motilidad de } \\
\text { Colecta }\end{array}$ & $\begin{array}{l}\text { Activación de } \\
\text { Motilidad }\end{array}$ & $\begin{array}{l}\text { Motilidad de } \\
\text { Colecta }\end{array}$ & $\begin{array}{l}\text { Activació } \\
\mathrm{n} \text { de } \\
\text { Motilidad }\end{array}$ \\
\hline 1 & 300 & 0 & 50 & 50 & 0 & 30 & 0 & 0 & 0 & 0 \\
\hline 2 & 350 & 0 & 0 & 90 & 0 & 90 & 0 & 90 & 0 & 0 \\
\hline 3 & 400 & 0 & 0 & 80 & 0 & 50 & 0 & 70 & 1 & 40 \\
\hline 4 & 450 & 0 & 0 & 90 & 0 & 80 & 0 & 80 & 0 & 20 \\
\hline 5 & 300 & 150 & 0 & 60 & 0 & 0 & 0 & 0 & 0 & 0 \\
\hline 6 & 350 & 150 & 0 & 70 & 0 & 0 & 0 & 0 & 0 & 0 \\
\hline 7 & 400 & 150 & 0 & 70 & 0 & 0 & 0 & 0 & 0 & 0 \\
\hline 8 & 450 & 150 & 0 & 60 & 0 & 0 & 0 & 0 & 0 & 0 \\
\hline
\end{tabular}

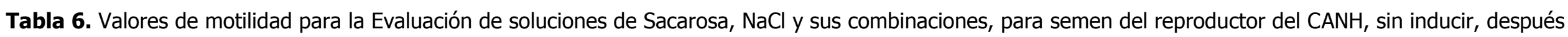
de una hora almacenado a temperatura ambiente.

\begin{tabular}{ccccccccccccc}
\hline No & Solución (mM) & \multicolumn{10}{c}{ MOTILIDAD* (\%) } \\
\cline { 3 - 12 } & & & & \multicolumn{10}{c}{ Replica 1 } & \multicolumn{1}{c}{ Réplica 2 } & \multicolumn{1}{c}{ Replica. 3 } & Replica. 4 & Replica. 5 \\
\cline { 3 - 12 } & Sacarosa & $\mathrm{NaCl}$ & $\mathrm{s} / \mathrm{H}_{2} \mathrm{O}$ & $\mathrm{c} / \mathrm{H}_{2} \mathrm{O}$ & $\mathrm{s} / \mathrm{H}_{2} \mathrm{O}$ & $\mathrm{c} / \mathrm{H}_{2} \mathrm{O}$ & $\mathrm{s} / \mathrm{H}_{2} \mathrm{O}$ & $\mathrm{c} / \mathrm{H}_{2} \mathrm{O}$ & $\mathrm{s} / \mathrm{H}_{2} \mathrm{O}$ & $\mathrm{c} / \mathrm{H}_{2} \mathrm{O}$ & $\mathrm{s} / \mathrm{H}_{2} \mathrm{O}$ & $\mathrm{c} / \mathrm{H}_{2} \mathrm{O}$ \\
& 300 & 0 & 0.00 & 0.00 & 0.00 & 0.00 & 0.00 & 0.00 & 0.00 & 0.00 & 0.00 & 0.00 \\
2 & 400 & 0 & 0.00 & 30.00 & 0.00 & 80.00 & 0.00 & 60.00 & 0.00 & 60.00 & 0.00 & 60.00 \\
3 & 450 & 0 & 0.00 & 0.00 & 0.00 & 0.00 & 0.00 & 0.00 & 0.00 & 0.00 & 0.00 & 0.00 \\
4 & 350 & 150 & 0.00 & 0.00 & 0.00 & 0.00 & 0.00 & 0.00 & 0.00 & 0.00 & 0.00 & 0.00 \\
5 & 400 & 150 & 0.00 & 60.00 & 0.00 & 0.00 & 0.00 & 0.00 & 0.00 & 0.00 & 0.00 & 0.00 \\
\hline
\end{tabular}

*Motilidad evaluada utilizando agua destilada, para activar la motilidad. 


\section{DISCUSIóN}

Los valores obtenidos en la inactivación de motilidad utilizando cloruro de sodio y bicarbonato, no tuvieron un efecto inactivador, por el contrario tuvieron un efecto en el control de la movilidad del semen, activando la motilidad para las muestras colectadas. Así también, en muchas especies el $\mathrm{Ca}$ tiene influencia en el $\mathrm{K}$ o en el $\mathrm{Na}$, lo cual constituye una señal de iniciación de la movilidad del semen. Estos resultados tendieron a coincidir con lo reportado por Hadi \& Cosson (2006), en relación a los efectos de iones y osmolalidad en la motilidad espermática in peces (ciprinidos y salmones). Así también, esto coincide con Martino (2006), reporta que los porcentajes de motilidad aumentan en un $20 \%$ en relación al semen activado con agua pura.

Se tiene a la actualidad varios estudios para criopreservación de semen para especies amazónicas. Sin embargo estos trabajos, difieren entre sí en el porcentaje de fertilización post descongelación de semen criopreservado. Tal como lo reporta Matino (2006), Maria et al. (2006), Fresneda et al (2004) y Navarro et al (2004).

El uso de glucosa, como crioprotector (relacionado a la capacidad de proteger al espermatozoide), en combinación con glicol de metilo para semen de Piaractus brachypomus, muestran altos valores (81\%), de motilidad en semen descongelado, como lo reporta Nascimento et al. (2010). Por lo tanto, puede ser usada para facilitar procedimientos de reproducción artificiales.

Para la especie $C$. macropomum, aún no se han reportado valores de osmolalidad, para conservar el semen inmótil y evitar la activación, de los espermatozoides, el cual va a influir en los porcentajes de motilidad al momento de la pos descongelación, de los lotes de semen crio preservados y por consiguiente aumentar la tasa de fertilización. Este trabajo aportaría el conocimiento de la utilización de sacarosa, que va a permitir mantener inactivos lotes de semen, antes de la criopreservación y poder trabajar con tranquilidad en el protocolo de criopreservación.

Dicho aporte en osmolalidad, son un punto crítico, para establecer protocolos de criopreservación, como lo reporta Cabrita et al. (2010), quienes resaltan la optimización en el uso del esperma congelado para la reproducción y el manejo de la selección, lo cual define la suceptibilidad de las muestras. Así mismo, el progreso en estas áreas podría contribuir con la standarizacion de los métodos y podría porveer una garantía en el mejoramiento y la seguridad del uso de un protocolo de criopreservación estandarizado, de semen para la acuicultura.

\section{CONCLUSIÓN}

Con los resultados del presente estudio, se puede concluir que la Sacarosa (400 mM.), influye manteniendo inactivo a los espermatozoides de Colossoma macropomum, al momento de la colecta, tanto de reproductores inducidos con Conceptal $®$ o sin inducir, manteniéndolos con el vigor de motilidad necesario, para el proceso de fecundación; sin embargo se puede aseverar que se puede mantener semen colectado en solución de Sacarosa $(400 \mathrm{mM})$, a temperatura ambiente por un tiempo de una hora. Por lo que este trabajo inicial permitirá el desarrollo de un protocolo de crio conservación para lotes de semen inmovilizados, obteniendo el tiempo suficiente para la preparación de las pajuelas y el congelamiento, en nitrógeno líquido.

\section{AGRADECIMIENTOS}

Al Proyecto cátedra CONCYTEC, IIAP e IRD Francia, por el financiamiento para la ejecución del presente estudio. Al personal profesional, técnico y practicantes de pregrado del CIQ-IIAP, por el apoyo en la ejecución del estudio.

\section{REFERENCIAS BIBLIOGRÁFICAS}

Cabrita E, Sarasquete C, Martínez-Paramo S, Robles V, Beira J, Pérez -Cerezales S, Herraéz M. 2010. Cryopreservation of fish sperm: applications and perspectives. J Appl Ichthyol 26: 623-635.

Carolsfeld J, Godinho H, Zaniboni E, Harvey B. 2003. Cryopreservation of sperm in Brazilian migratory fish conservation. J Fish Biol 63: 472-489.

Chereguini O, Fernández P, Rasines I. 1992. Adaptación de la técnica de críopreservación de esperma para el Rodaballo (Scohthalmus maximus) y Besugo (Pagellus bogaraveo). Instituto español de Oceanografía 117: 1-11. 
Ciereszko A, Dabrowski K. 1993. Estimation of sperm concentration of rainbow trout, whitefish and yellow perch using a spectrophotometric technique. Aquaculture 109: 367-73.

Fresneda A, Lenis G, Agudelo E, Olivera M. 2004. Espermiación inducida y crioconservación de semen de cachama blanca (Piaractus brachypomus). Rev Col Cienc Pec 17: 46-52.

Hadi S, Cosson J. 2006. Sperm motility in fishes. (II) Effects of ions and osmolality: A review. Cell Biology International 30 (9): 1-14.

Krone A, Wittbrodt J. 1997. A simple and reliable protocol for cryopreservation of Medaka (Oryzias latipes) spermatozoa. The Fish Biology Journal Medaka 9: 4748.

Leung K, Jamieson B. 1991. Live preservation of fish gametes. En: Jamiesson BGM, editor. Fish evolution and systematics: Evidence from spermatozoa. Cambridge: University Press: 245-69 pp.

Liu Q, Li J, Zhang S, Ding F, Xu X, Xiao Z, Xu S. 2006. An Efficient Methodology for Cryopreservation of Spermatozoa of Red Seabream, Pagrus major, with 2-mL Cryovials. Journal of the world Aquaculture Society 37 (3): 289-297.

Maria A, Viveiros A, Freitas R, Oliveira A. 2006. Extenders and cryoprotectants for cooling and freezing of piracanjuba (Brycon orbignyanus) semen, an endangered Brazilian teleost fish. Aquaculture 260: 298-306.

Martino G. 2006. Primeros ensayos sobre críopreservación de semen de Cachama Colossoma macropomun y Morocoto Piaractus brachypomus. IV Congreso Iberoamericano Virtual de Acuicultura. 152-158 pp.
Murgas L, Gualhanone A, Silva M, Mello C, Freitas R, Zangeronimo M. 2001. Calidad seminal del pez piracanjuba (brycon orbignyanus) post-descongelación. An Vet Murcia 17: 3-10.

Nascimento A, Maria A, Pessoa N, Carvalho M, Viveiros A. 2010. Out-of-season sperm cryopreserved in different media of the Amazonian freshwater fish pirapitinga (Piaractus brachypomus). Animal Reproduction Science 118: 324-329.

Navarro O, Velasco Y, Casallas P. 2004. Evaluación de cincro protectores para la crioconservación de semen de Cachama Blanca (Piaractus brachypomus). Rev Col Cienc Pec 17: 53-59.

Nizio A., Costa H, Falanghe P. 2011. Protocolo para Criopreservação do Sêmen de

Tambaqui (Colossoma macropomum). Comunicado técnico 112. MInistério da Agricultura. Pecuária e Abastecimento. $1^{\circ}$ Ed. 1-8 pp.

Otemé J, Nuñez J, Kouassi C, Agnese J, Hem S. 1996. Testicular structure, spermatogenesis and sperm cryopreservation of the african catfish Heterobranchus longifilis (Valenciennes, 1840). Aquaculture Research 27 (11): 805-813.

Ribeiro R, Godinho H. 2003. Criopreservação do semen testicular do teleósteo piau-açu Leporinus macrocephalus. Arq Bras Med Vet Zootec 55(1): 1-7.

Valdebenito L. 2008. Terapias hormonales utilizadas en el control artificial de la madurez sexual en peces de cultivo: una revisión. Arch med vet 40 (2): 115-123. 